\title{
Flame Retardant Finish of Silk Fabric with Dimethyl Phosphonate Doped Silica Sol
}

\author{
Qiang-Hua ZHANG ${ }^{1}$, Wei ZHANG ${ }^{1}$, Guo-Qiang $\mathrm{CHEN}^{1}$, Tie-Ling XING ${ }^{2, a,{ }^{*}}$ \\ ${ }^{1}$ National Engineering Laboratory for Modern Silk, College of Textile and Engineering, Soochow \\ University, Suzhou, 215123, China \\ ${ }^{2}$ Jiangsu HuaJia Group, Suzhou, 215000, China \\ axingtieling@suda.edu.cn \\ ${ }^{*}$ Corresponding author
}

Keywords: Organic Phosphorus Flame Retardant, Sol-gel Method, Silica Sol, Silk Fabric, Thermo Stability.

\begin{abstract}
In this paper, a dimethyl phosphonate doped (P-doped) silica sol was prepared from a tetraethyl silicate inorganic precursor and applied to silk fabric as a flame retardant finish through the sol-gel process. The X-ray photoelectron spectroscopy (XPS) analysis and scanning electron microscope (SEM) images indicated a phosphorus-containing silica network successfully formed on the silk fabric surface. The flammability of the treated silk fabric was investigated by the limiting oxygen index (LOI) and vertical combustion test. The results indicated that the treated silk fabric were flame retardant with a higher limiting oxygen index (LOI) and shorter damage length. Moreover, micro calorimeter combustion testing and thermal gravimetric analysis indicate $50 \%$ of P-doped silica sol treated silk fabric exhibited a lower heat release rate and higher char residue, which demonstrates a synergistic effect between the silica and phosphorus.
\end{abstract}

\section{Introduction}

The flame retardance of a textile is rather important for both researchers and consumers because textiles' igniting during a fire can cause damage to property and life. According to Great Britain fire statistics from April 2012 to March 2013, 76\% of fire-related fatalities occurred in dwelling fires, and textiles along with upholstery and furnishings accounted for nearly $60 \%$ of deaths ${ }^{[1]}$. Indeed, studying textile flame retardancy began very early, and most currently used flame retardant chemicals were developed during from 1950-1980. However, little work has occurred in the last decade due to increasing environmental sustainability, chemical toxicological acceptability, performance and cost concerns ${ }^{[2]}$.

The sol-gel process, an emerging material surface modification technology, is mainly based on the hydrolysis and condensation of semi-metal alkoxides. The sol-gel method has been widely used to create functionalized textiles ${ }^{[3]}$, such as antimicrobial, water repellent and super hydrophobic textiles because it yields new materials with a high degree of molecular homogeneity and potentially extraordinary physical and chemical properties ${ }^{[4]}$. In recent years, the sol-gel method has been increasingly used and reportedly exhibits great potential for flame retardancy ${ }^{[5]}$.

The high nitrogen and sulfur content of silk fabric makes it less flammable than other fibers. However, it still cannot satisfy the strict demand for flame-retardant textiles. In this paper, silk fabric was treated with a P-doped silica sol using dimethyl phosphonate as a flame retardant additive via the sol-gel method. Flammability, combustion performance under thermal radiation and thermo stability tests were performed to comprehensively characterize the flame retardant properties. The surface morphologies and elemental composition for the finished fabrics were also presented. 


\section{Experimental}

\section{Material and Reagents}

Silk fabric (weight: $36 \mathrm{~g} / \mathrm{m} 2$, density (ends/cm): 50×45 (warp $\times w e f t))$ was supplied by Suzhou Huasi Silk Printing \& Dyeing Co., LTD (Suzhou, China). Tetraethyl silicate (TEOS), the inorganic precursor, and dimethyl phosphonate (DP), the flame retardant additive, were both purchased from Sinopharm Chemical Regent Co., Ltd. Ethanol (EtOH) and hydrochloric acid $(\mathrm{HCl})$ were purchased from China Sun Specialty Products Co., Ltd. All reagents were analytically pure.

\section{Preparation of Flame Retardant Sol}

The flame retardant sol was synthesized via the sol-gel method. The pure silica sol was prepared as follows: $3.6 \mathrm{ml}$ distilled water was acidified with $1.4 \mathrm{ml}$ hydrochloric acid and added dropwise to a $44.7 \mathrm{ml}$ tetraethyl silicate and $35.0 \mathrm{ml}$ ethanol solution while stirring at room temperature. This solution was stirred for 3 hours at $70^{\circ} \mathrm{C}$ and aged for 2 days. The molar ratio for the reagents was 1:3:1:0.085 for TEOS: EtOH: $\mathrm{H}_{2} \mathrm{O}: \mathrm{HCl}$. For the P-doped silica sol, dimethyl phosphonate, used as a flame retardant, was added to the tetraethyl silicate before the hydrolysis. To investigate the synergistic effect between tetraethyl silicate and dimethyl phosphonate, different mole dimethyl phosphonate fractions (namely, 0, 10, 20, 30, 40 and 50\% with respect to the sum of TEOS and DP) were employed.

\section{Modification of Silk Fabric with Prepared Sols}

According to the padding \& curing modification process, the silk fabric was first impregnated with the prepared sols and squeezed for a $100 \%$ pickup via a padder with a $0.4 \mathrm{~kg} / \mathrm{cm} 2$ nip pressure. The samples were then dried at $80^{\circ} \mathrm{C}$ for $3 \mathrm{~min}$ and immediately cured at $120^{\circ} \mathrm{C}$ for $6 \mathrm{~min}$. All samples were conditioned under standard atmospheric conditions $\left(25 \pm 2{ }^{\circ} \mathrm{C} ; 65 \%\right.$ humidity $)$ for $48 \mathrm{~h}$ before testing.

\section{Characterization and Measurements}

Morphology of the samples was observed by a Hitachi TM3030 Desktop Scanning Electron Microscope (SEM) at an acceleration voltage of $3 \mathrm{kV}$ under vacuum condition. The elemental binding state was measured via X-ray photoelectron spectroscopy (XPS, Axis Ultra HAS, Kratos). The step size for the high-resolution scan was $0.1 \mathrm{eV}$, and the pressure in the analysis chamber was maintained at $4.0 \times 10^{-9} \mathrm{pa}$.

The limiting oxygen index (LOI) was measured by LCK-09 Oxygen index measuring instrument according to ASTM D2863. The vertical combustion test was measured by LYF-26 vertical combustion instrument, and the fabric bottom $(300 \mathrm{~mm} \times 80 \mathrm{~mm})$ was exposed to a $40 \pm 2 \mathrm{~mm}$ high flame for $12 \mathrm{~s}$ to calculate the damage length, repeating 4times for each sample. Micro calorimeter combustion (MCC) instrument was used to test the sample combustion properties. The samples $(\sim 5 \mathrm{mg})$ were heated from room temperature to $700^{\circ} \mathrm{C}$ under nitrogen flow at $80 \mathrm{~cm}^{3} / \mathrm{min}$ with a linear heating rate of $1^{\circ} \mathrm{C}$. The gaseous pyrolysate mixture in the pyrolyser was mixed with a $20 \mathrm{~cm}^{3} / \mathrm{min}$ stream of oxygen before combusting in a $900^{\circ} \mathrm{C}$ furnace for $10 \mathrm{~s}$. The thermo stability the silk fabrics were performed with a 2960 SDT 290 TA instruments. The temperature was set from $50^{\circ} \mathrm{C}$ to $700^{\circ} \mathrm{C}$ at a rate of $10^{\circ} \mathrm{C} / \mathrm{min}$ and nitrogen atmosphere was selected.

In addition, the add-on values of the samples (Table 1) were calculated according to equation 1 :

$$
\text { Add-on }=w_{2}-w_{1} / w_{1} \times 100 \%
$$


where $w_{1}$ and $w_{2}$ is the weight of silk fabric before and after modification, respectively. The treated fabrics were washed for $60 \mathrm{~min}$ using a $5 \mathrm{~g} / \mathrm{l}$ soap-flake at $60^{\circ} \mathrm{C}$ before testing to understand the modified silk fabric durability.

\section{Results and Discussions}

\section{Surface Morphology and Chemical Composition Characterization}

Figure 1 shows the surface morphology of the treated (pure and 50\% P-doped) and control silk fabrics. It can be seen from the figure that the control sample surface was clean and smooth with clear boundaries between each filament (a), while the treated fabric surface were fuzzy and covered by the coatings ( $b$ and $c$ ). The char residue after heating the treated samples (e and f) to $700^{\circ} \mathrm{C}$ retained a complete and stable structure in contrast to the control (d). This result indicates the silicon network successfully formed on the silk fabric surface after the treatment and could guarantee the structural integrality at high temperatures.
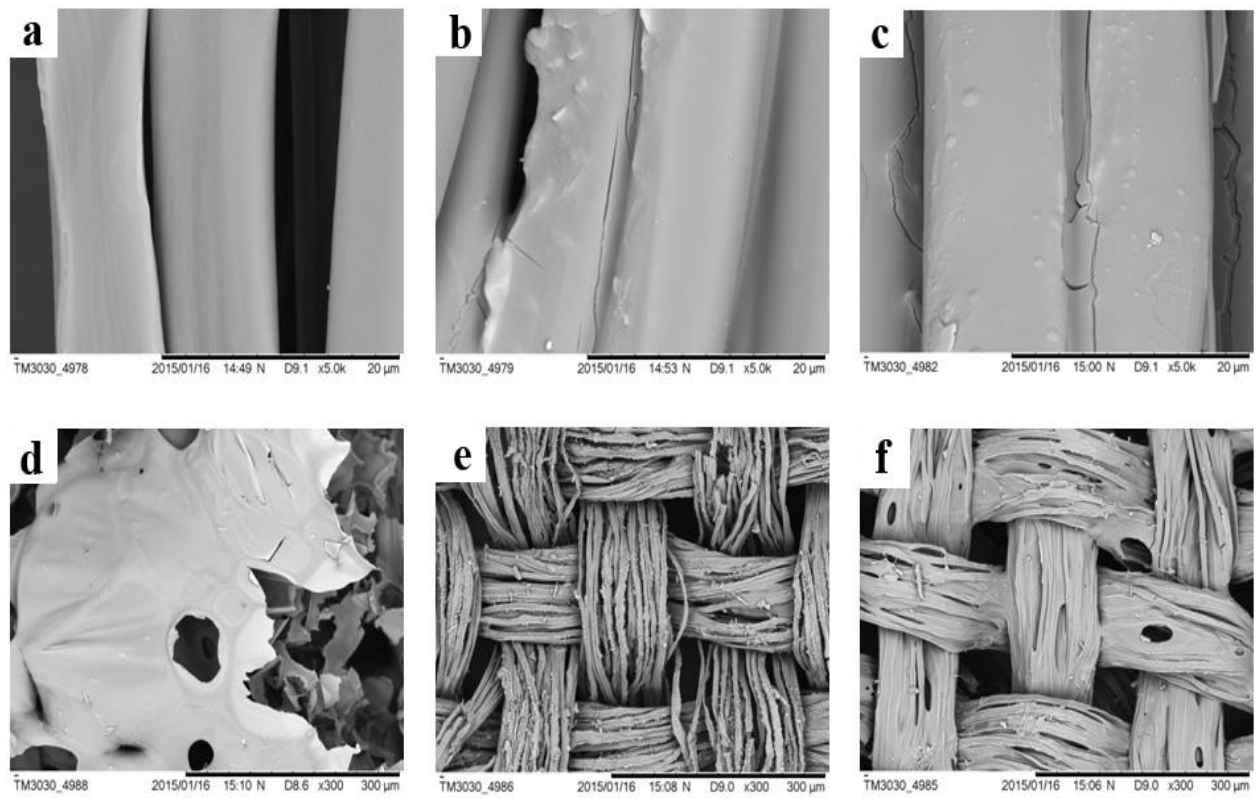

Fig.1 SEM Images of Silk Fabric for Control (a), Pure Silica Sol Treated (b) and 50\% P-doped Silica Sol Treated Sample (c) and Char Residue for the Control (d), Pure Silica Sol Treated (e) and $50 \%$ P-doped Silica Sol Treated Sample (f), respectively

The surface composition for the samples were investigated by XPS. Figure 2 shows a typical survey for the three samples. The distinctive silica peak was present for the treated silk fabrics (b and c) but not the control (a). In particular, according to the literature [6,7], the Si $2 p$ peaks from the -Si-O-Si- chemical bond appeared with a binding energy of $98 \mathrm{eV}$ for pure silica sol treated silk fabric and $100 \mathrm{eV}$ for the $50 \% \mathrm{P}$-doped silica sol treated silk fabric. The $\mathrm{SiO}_{2}$ films formed on the surface of the treated silk fabrics via the sol-gel method. The Si 2 s peaks from the -Si-O-Cchemical bond exhibited a binding energy of $149 \mathrm{eV}$ for the pure silica sol treated silk fabric and $151 \mathrm{eV}$ for the P-doped silica sol treated silk fabric, which confirmed the silica films were grafted to the sample surface. Furthermore, the P $2 p$ peak with a binding energy of $130 \mathrm{eV}$ for the $50 \%$ P-doped silica sol treated silk fabric indicated phosphorus was deposited on the silk fabric via silica sol doping ${ }^{[7]}$. 


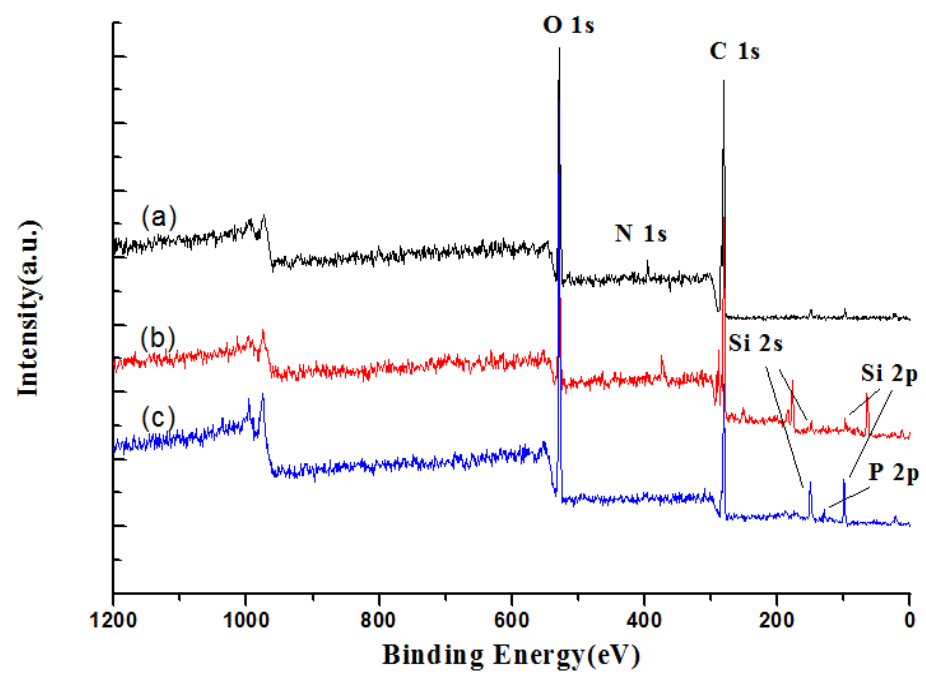

Fig.2 XPS Survey Spectra of (a) Control (b) Pure Silica Sol Treated and (c) 50\% P-doped Silica Sol Treated Sample

\section{Flammability Test}

The LOI and vertical combustion experimental results are shown in Table 1.

Tab.1 Flammability Test for Treated and Untreated Silk Fabric

\begin{tabular}{|c|c|c|c|c|c|}
\hline \multirow[t]{2}{*}{ Code } & \multirow{2}{*}{$\begin{array}{c}\text { Concentration of dimethyl } \\
\text { phosphonate } \\
(\%)\end{array}$} & \multirow{2}{*}{$\begin{array}{l}\text { Add-on } \\
(\%)\end{array}$} & \multicolumn{2}{|c|}{ LOI (\%) } & \multirow{2}{*}{$\begin{array}{l}\text { Damage length } \\
(\mathrm{mm})\end{array}$} \\
\hline & & & $\begin{array}{c}\text { Before } \\
\text { washing }\end{array}$ & $\begin{array}{c}\text { After } \\
\text { washing }\end{array}$ & \\
\hline CONTROL & --- & --- & 24.2 & 24.2 & 230 \\
\hline TEOS & 0 & 23.17 & 26.5 & 26.3 & 121 \\
\hline TEOS 10P & 10 & 22.56 & 26.9 & 26.3 & 132 \\
\hline TEOS 20P & 20 & 26.74 & 27.4 & 26.9 & 141 \\
\hline TEOS 30P & 30 & 24.41 & 27.4 & 26.5 & 122 \\
\hline TEOS 40P & 40 & 26.07 & 28.2 & 26.8 & 112 \\
\hline TEOS 50P & 50 & 30.02 & 33.1 & 27.7 & 98 \\
\hline
\end{tabular}

The LOI values were higher for the treated silk fabrics than the control sample, which means the flame retardance of silk fabric was improved after treating with the prepared sols. More specifically, the LOI value for the pure silica sol treated silk fabric was $26.5 \%$, a $9.5 \%$ increase over the control sample $(24.2 \%)$. The LOI value for the P-doped silica sol treated silk fabric increased relatively slowly for a P concentration below 30\%. However, increasing the dimethyl phosphonate concentration to $50 \%$ gave the treated silk fabric an optimal flame retardance with a $33.1 \%$ LOI because both silica and phosphorus are flame retardant elements. The sol-gel treatment forms a nonflammable silica network on the treated silk fabric surface, which prevented the flames from spreading. the dimethyl phosphonate, a phosphorus-based flame retardant, exhibits a significant flame retardant effect on the fabric by generating phosphorous acid that dilutes the flammable gas from combustion and improves the flame retardance of the treated fabric. Notably, the LOI values for all of the silk fabrics treated with a P-doped silica sol remained above $26.0 \%$ after home laundering once, which indicates the flame retardance has a certain durability. Furthermore, the damage length was much shorter for all of the treated silk fabrics than the control sample, which indicates reduced vertical combustion.

\section{Combustion Performance}

Table 2 shows the heat release data for the silica sol treated and control silk fabrics during MCC test and Figure 3 shows the heat release curves versus temperature for the test samples. 
Tab.2 MCC Data for Treated and Untreated Silk Fabric

\begin{tabular}{|c|c|c|c|c|c|}
\hline Sample & $\begin{array}{c}\text { HRC } \\
(\mathrm{J} / \mathrm{g} \cdot \mathrm{K})\end{array}$ & $\begin{array}{c}\mathrm{pHRR} \\
(\mathrm{W} / \mathrm{g})\end{array}$ & $\begin{array}{c}\text { THR } \\
(\mathrm{kJ} / \mathrm{g})\end{array}$ & $\begin{array}{c}\mathrm{T}_{\max } \\
\left({ }^{\circ} \mathrm{C}\right)\end{array}$ & $\begin{array}{c}\text { Char residue } \\
(\%)\end{array}$ \\
\hline CONTROL & 109 & 97.3 & 12.3 & 296.4 & 20.60 \\
\hline TEOS & 122 & 111.9 & 10.5 & 313.7 & 33.96 \\
\hline TEOS 10P & 115 & 104.6 & 8.7 & 309.7 & 32.20 \\
\hline TEOS 20P & 103 & 93.8 & 7.6 & 306.5 & 33.60 \\
\hline TEOS 30P & 101 & 92.7 & 7.4 & 305.2 & 34.93 \\
\hline TEOS 40P & 99 & 90.8 & 7.5 & 304.2 & 36.40 \\
\hline TEOS 50P & 78 & 71.5 & 6.3 & 293.5 & 45.60 \\
\hline
\end{tabular}

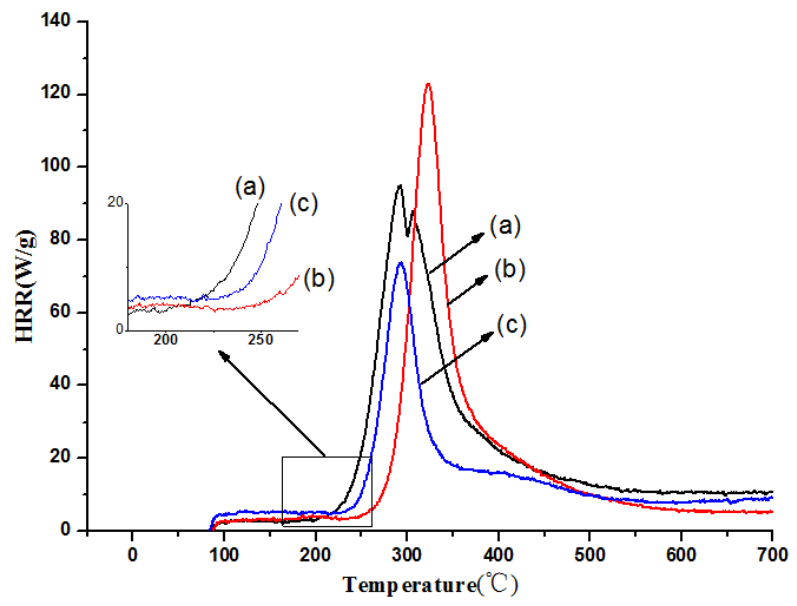

Fig.3 HRR Curves of (a) Control (b) Pure Silica Sol Treated and (c) 50\% P-doped Silica Sol Treated Sample

Table 2 shows the peak heat release rate (pHRR) decreased with increasing $\mathrm{P}$ concentration in the prepared sol. Compared to the pure silica sol treated silk fabric, the pHRR for the silk fabric treated using a silica sol with $50 \% \mathrm{P}$ content decreased from 111.9 to $71.5 \mathrm{~W} / \mathrm{g}$. However, compared to the control sample, the pHRR of the silica sol treated silk fabric did not decrease using pure silica or a lower P content (below 20\%). The same pHRR trend was observed for the heat release capacity (HRC) value, which was obtained by dividing the maximum specific heat release rate (SHRR) by the heating rate for the test $8^{[8]}$. The silica layer could only act as heat insulation on the treated fabric surface and prevent heat release to some extent because a low P content only weakly prevents the thermal degradation of the sample. When the heat under the insulation layer reached a limit, all of the accumulated heat broke through the layer and was released, which makes the maximum heat release rate and heat release capacity larger than the control sample. However, when the P content increased from $20 \%$ to $50 \%$, the thermal degradation of the silica sol treated silk fabric changed significantly, which made the heat insulation from the silica layer stable enough to endure the heat release, which decreased the pHRR and HRC. As depicted in Figure 6, the treated (pure and 50\% P-doped) silk fabric began decomposing at $250^{\circ} \mathrm{C}$ (b) and $230^{\circ} \mathrm{C}$ (c), which is higher than for the control sample, $200^{\circ} \mathrm{C}$ (a), as shown by the rapid increase in HRR and dramatic decomposition at lower temperatures. Furthermore, the treated silk fabric exhibited a lower total heat release (THR) and higher percent char residue than the control sample. These results indicated improved combustion properties for the treated silk.

\section{Thermal Stability}

The TG curves for the treated (pure and 50\% P-doped) and control silk fabrics are shown in Figure 4. 


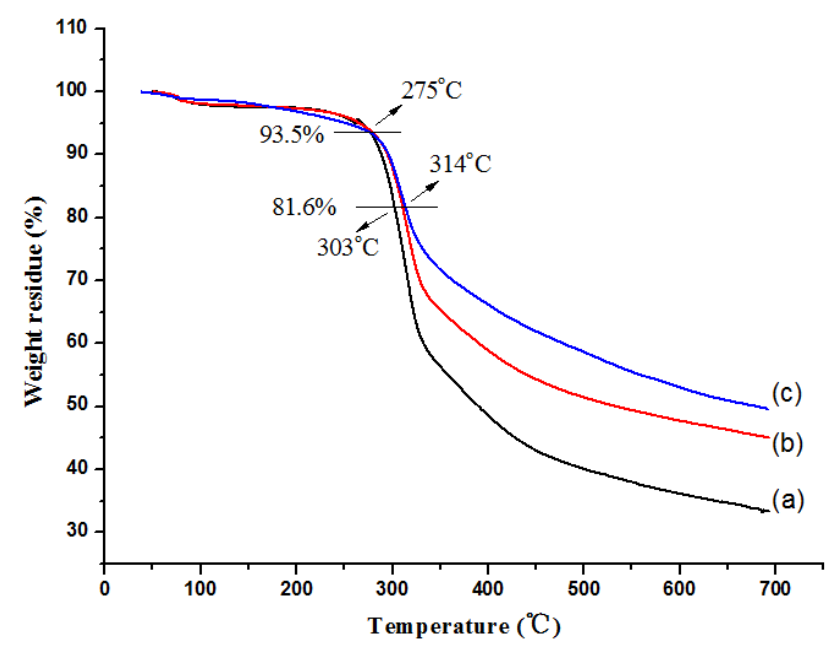

Fig.4 TG Curves of (a) Control (b) Pure Silica Sol Treated and (c) 50\% P-doped Silica Sol Treated Sample

The three curves exhibited similar weight loss behavior below $275^{\circ} \mathrm{C}$ due to water loss. The silk fabric began to seriously degrade with a major weight loss from $280^{\circ} \mathrm{C}$ to $500^{\circ} \mathrm{C}$. Notably, the temperature for the treated silk fabric for any given weight was slightly higher than for the control sample. For example, when the weight residue decreased to $81.6 \%$, the temperature of the treated silk fabric was nearly $314^{\circ} \mathrm{C}$ while the control sample was $303^{\circ} \mathrm{C}$. This result indicated the thermal-degradation temperature was improved for the treated silk fabric. Additionally, increasing the temperature stabilized the weight loss rate for the silk fabric and a steady char residue was obtained at nearly $700^{\circ} \mathrm{C}$. As shown in the figure, the char residue weight was much lower for the control than the treated samples, i.e., $33.30 \%$ for the control sample and $45.10 \%$ and $49.65 \%$ for the pure and 50\% $\mathrm{P}$ doped silica sol treated silk fabrics, respectively. Indeed, according to the report, individual phosphorus flame retardants could not improve the thermal stability. Phosphorus flame retardants act as a condensed form of phosphoric acid and polyphosphoric acid during thermal decomposition and cannot gain char residue but only dehydrates carbon-containing materials, which accelerates oxidation ${ }^{[9]}$. Therefore, the increased char residue for the $50 \%$ P-doped sample could evidence a synergistic effect between the silica and phosphorus, that is, the phosphorus-containing component could promote the material dehydrating to carbon at high temperatures, and the silica layer could then improve the produced carbon stability.

\section{Summary}

A nano-scaled silica sol system was prepared and applied as a flame retardant finish on silk fabric. The results indicated the flame retardance of the silk fabric was improved after treating with pure and dimethyl phosphonate doped silica sols. The treated silk fabric reached the optimal flame retardant effect (33.1\% of the LOI compared to $24.2 \%$ for the control sample) and superior thermal stability when treated with a $50 \%$ P doped silica sol.

\section{Acknowledgement}

This work is supported by the Priority Academic Program Development of Jiangsu Higher Education Institutions (PAPD), Natural Science Foundation of Jiangsu Province (BK20151242) and sponsored by Qing Lan Project. 


\section{References}

[1] Fire Statistics: Great Britain April 2012 to March 2013.Fire \& Rescue Statistical Release: Great Britain April 2012 to March 2013: www.gov.uk/dclg

[2] Horrocks AR. Flame retardant challenges for textiles and fibres: New chemistry versus innovatory solutions. Polymer Degradation and Stability 2011. 96:377-392.

[3] Mahltig B, Haufe H, Böttcher H. Functionalisation of textiles by inorganic sol-gel coatings. Journal of Materials Chemistry 2005. 15: 4385-4398.

[4] Alongi J, Malucelli G. State of the art and perspectives on sol-gel derived hybrid architectures for flame retardancy of textiles. Journal of Materials Chemistry 2012. 22: 21805-21809.

[5] Yaman N. Preparation and flammability properties of hybrid materials containing phosphorus compounds via sol-gel process. Fibers and Polymers 2009. 10(4): 413-418.

[6] Lin J, Wu X, Zheng C, Zhang PP, Huang BW , Guo NH , Jin LYZ. Synthesis and properties of epoxy-polyurethane/silica nanocomposites by a novel sol method and in-situ solution polymerization route. Applied Surface Science 2014. 303: 67-75.

[7] Kačiulis S, Mattogno G, Pandolfi L, Cavalli M, Gnappi G, Montenero A. XPS study of apatite-based coatings prepared by sol-gel technique. Applied Surface Science 1999. 151: 1-5.

[8] Chen LJ, Tai QL, Song L, Xing WY, Jie GX, Hu Y. Thermal properties and flame retardancy of an ether-type UV-cured polyurethane coating. Express Polymer Letters 2010. 4: 539-550.

[9] Alongi J, Ciobanu M, Malucelli G. Novel flame retardant finishing systems for cotton fabrics based on phosphorus-containing compounds and silica derived from sol-gel processes. Carbohydrate Polymers 2011. 85: 599-608. 